Bond University

Research Repository

\title{
Focus on sharing individual patient data distracts from other ways of improving trial transparency
}

Hoffmann, Tammy; Glasziou, Paul; Beller, Elaine; Goldacre, Ben; Chalmers, lain

Published in:

BMJ (Online)

DOI:

10.1136/bmj.j2782

Licence:

Unspecified

Link to output in Bond University research repository.

Recommended citation(APA):

Hoffmann, T., Glasziou, P., Beller, E., Goldacre, B., \& Chalmers, I. (2017). Focus on sharing individual patient data distracts from other ways of improving trial transparency. BMJ (Online), 357, [j2782].

https://doi.org/10.1136/bmj.j2782

\section{General rights}

Copyright and moral rights for the publications made accessible in the public portal are retained by the authors and/or other copyright owners and it is a condition of accessing publications that users recognise and abide by the legal requirements associated with these rights.

For more information, or if you believe that this document breaches copyright, please contact the Bond University research repository coordinator 


\section{Is the focus on Individual Patient Data sharing a distraction from other ways of}

increasing trial transparency? Focusing on making IPD available is not a cost-effective way of increasing trial transparency.

Tammy Hoffmann $\mathrm{PhD}^{1}$

Paul Glasziou $\mathrm{PhD}^{1}$

Elaine Beller MAppStat ${ }^{1}$

Ben Goldacre MRCPsych ${ }^{2}$

Iain Chalmers DSc ${ }^{3}$

${ }^{1}$ Centre for Research in Evidence-Based Practice, Faculty of Health Sciences and Medicine, Bond University, Gold Coast, Australia

${ }^{2}$ Centre for Evidence-Based Medicine, University of Oxford, UK

${ }^{3}$ James Lind Initiative, Oxford, UK

Corresponding author:

Prof Tammy Hoffmann, Centre for Research in Evidence-Based Practice, Faculty of Health Sciences and Medicine, Bond University, Gold Coast, Australia.

thoffmann@bond.edu.au

Tel: +61 755955522 


\section{Introduction}

The International Committee of Medical Journal Editors (ICMJE) has issued the latest of a series of calls promoting the sharing of individual patient data (IPD) from randomised trials. ${ }^{1}$ Valuable as IPD sharing can be ${ }^{2}$ discussion about it has hijacked the broader conversation about data sharing and trial transparency. Numerous prominent editorials and commentaries have discussed data sharing passionately, ${ }^{3-5}$ however the debate is mostly focused on IPD. For example, we analysed all 76 articles published in the six leading general medical journals that had "data" and "sharing" in their title and were about clinical trials. In 64 (84\%) articles, the content was focussed on IPD and did not mention any of the other components of trial transparency (see eAppendix 1). While trial transparency is a major impetus of data sharing, sharing just IPD does not achieve this. Trial transparency requires a data sharing 'package', which begins with trial registration and contains other elements such as protocols, summary results, and trial materials.

Because IPD considerations have dominated the trial transparency agenda, much discussion has focused on the complexities and practical problems associated with IPD sharing and the processes and systems needed for responsible data sharing. ${ }^{5-8}$ However, many of the data sharing activities that are needed for trial transparency are not complex. We believe that trying to solve the complex issues around IPD availability should not eclipse or distract from a more pressing problem: the unavailability of even summary data and protocols from all controlled trials. Current estimates are that around $85 \%$ of research is avoidably "wasted" due to design flaws, poor conduct, nonpublication, and poor reporting. ${ }^{9}$ Focusing efforts and attention on making IPD accessible might paradoxically exacerbate this waste in research. We examine simpler and more cost-efficient activities that should be prioritised. These are summarised in Figure 1, and expand upon a previously-published trial reporting system. ${ }^{10}$

\section{Priorities}

\section{Prospective trial registration}

About one in two trials is never published. ${ }^{11}$ In 2005 the ICMJE introduced prospective registration as an attempt to minimise selective reporting. However many trials are still not prospectively registered, even those published in high-impact journals, ${ }^{12}$ and fewer than half of the journals that publish reports of trials enforce this requirement. ${ }^{13}$ As well as the problems of selective publication of trials and outcomes, unregistered trials, or those registered after the completion date, tend to yield larger estimates of treatment effects than those registered prior to completion. ${ }^{14}$ 
Trial registration is simple and inexpensive, and uses existing systems, such as ClinicalTrials.gov. The AllTrials campaign has been championing trial registration for all trials, as well as calling for summary results, and a full report (full methods, analyses, and results) to be publicly available, but the campaign has stated explicitly "We do not call for individual patient data to be made publicly available". ${ }^{15}$ The campaign has also highlighted ways in which trial registration could be improved. Although the World Health Organisation has fostered the development of an international standard for Trial Registration Data, ${ }^{16}$ minimum requirements are neither sufficient nor enforced.

\section{Summary results reported}

In 2007, ClinicalTrials.gov began requiring, as part of the Food and Drug Administration Amendments Act (FDAAA) that summary results of certain categories of trials (for example, trials of currently approved medicines, with at least one site in the United States (US), or conducted as part of a marketing approval application) be submitted within 12 months of the completion of data collection, whether or not the trial had been formally published in a journal. The information required includes tabular summaries of participant flow, baseline characteristics, pre-specified outcome measure values and adverse events by study arm or comparison group, and statistical analyses. However, subsequent audits have revealed poor attention to these requirements, with only about one trial in five complying. ${ }^{17}$ Furthermore, although the US Food and Drug Administration is entitled to impose fines of $\$ 10,000$ a day for non-compliance, penalties have never been levied. In September 2016, partly in response to low compliance and to remove ambiguity about the requirements, a final rule to the earlier FDAAA was announced. ${ }^{18}$ At the same time, the US National Institutes of Health $(\mathrm{NIH})$ issued a policy requiring all NIH-funded trials (including of interventions that are not covered by the FDAAA requirements, such as non-drug interventions) to submit registration and summary results to ClinicalTrials.gov. Compliance with this NIH policy and clarification of the FDAAA rule will need evaluation.

Posting a summary of trial results enables key information to be publicly and promptly available, including to those endeavouring to keep systematic reviews up to date. Indeed, information on participant flow, efficacy, and adverse events in trials is reported more promptly and in a higher proportion of trials in trial registries than in most reports published in journals. ${ }^{19,20}$ It is reasonably simple to do, requires a similar format to that required for publication in a journal article, can utilise existing platforms, and is not regarded as prior publication by the ICMJE. Pfizer has estimated that it takes between 5 and 60 hours of person-time to post a summary of the results of a completed trial to ClinicalTrials.gov. ${ }^{21}$ Assuming an average of 40 hours at $\$ 50$ per hour, the cost per trial would be 
about US $\$ 2,000$. This is a trivial sum in the context of cost estimates of US $\$ 42,000$ per participant for running a trial. ${ }^{22}$

Despite the benefits to trial transparency and the comparatively small time investment, ClinicalTrials.gov is currently the only public registry that has data fields to accept summary results for any registered trial. The European Clinical Trials Database also began requiring summary results in July 2014, but only accepts results of trials of drugs that are within the regulatory scope of the European Medicines Agency. It is too early to assess compliance with this requirement.

\section{Trial protocol available}

The minimum details currently required by trial registries are insufficient to enable confident interpretation and use of trial results in clinical practice. Good trial protocols facilitate full reporting and proper conduct of trials, ${ }^{23}$ however, they are not publicly available for most trials.

Inclusion of protocols in trial registries would be ideal, but this is currently rare. New regulations in the $\mathrm{US}^{24}$ require that those conducting trials of drugs and devices (but not other non-drug interventions) submit a copy of the protocol and statistical analysis plan for public posting. Although this requirement is a welcome development, it only applies to a proportion of the world's trials and it remains to be seen whether it will be enforced. Some registries allow protocols to be uploaded as PDFs (for example, the ISRCTN registry, and from June 2017, ClinicalTrials.gov will allow protocols in English), and others allow links to protocols published in journals or posted on other sites (for example, those for trials funded through the NIHR Health Technology Assessment Programme), but most registries do not offer either of these options.

\section{Other trial materials}

There are many trial materials that are important for interpreting, using, and replicating trials, however this information is rarely included in publications or otherwise made public. This includes: patient and investigator information (such as consent forms and trial information sheets), statistical analysis plans, blank case report forms, and reproducible descriptions of measurements and interventions. While ideally these materials should be included in protocols, they usually are not. Additionally, after the protocol is complete and the trial has commenced, modifications can occur to some of this information (such as intervention details and data analysis plans) and these modifications and updated materials should be available. 
As an example of the consequences of missing trial materials, consider adequate descriptions of the interventions assessed in trials. Without full details, interventions shown to be useful cannot be taken up by health professionals, patients, other researchers, and policy makers. ${ }^{15}$ Most analyses have found that over half of the studies examined have incomplete intervention descriptions (with intervention materials the most frequently missing component), ${ }^{26,27}$ and these and other trial materials are generally hard to access, even on request. ${ }^{26,28}$ The result is that studies supported by the public and to which patients have contributed are unusable and not replicable by others, with the entire trial investment becoming a sunk-cost. Another example of why access to other types of trial materials can be important is the need for transparency of consent forms and patient information sheets when participants who are assigned to control groups appear to have received care below accepted standards.

Initiatives to improve access to additional materials have included guidance on descriptions of interventions ${ }^{25}$ and public sharing platforms to facilitate sharing of trial materials, ${ }^{29}$ but these developments have not yet received mainstream support. As with summary results, trial materials should be publicly available after trial completion, yet discussion is needed to agree how to achieve this. Many registries do not accept PDF uploads, only some registries allow web links to be provided, most trials do not have websites, and links to journal online supplementary materials and other websites are too often unreliable.

\section{Clinical Study Reports}

For some trials (usually for interventions which require regulation or licensing), clinical study reports (CSR) are produced as the full trial report. CSRs contain many of the other elements of the trial transparency 'package' that have been described in this article (such as detailed protocols, statistical analysis plans, and efficacy and safety evaluations). ${ }^{30}$ As such, where CSRs exist, they can make an important contribution towards trial transparency and should be made publicly available, even if it is without the IPD that they sometimes contain.

\section{All trials for any and all interventions}

Calls to improve access to trial data tend to focus on drug, biologic, and medical device trials, probably because regulatory approval is required for these interventions. Despite views to the contrary, ${ }^{31}$ these initiatives apply equally to trials of non-regulated interventions. About $40 \%$ of published reports of randomised trials are concerned with the effects of non-regulated interventions. ${ }^{32}$ Furthermore, trials of non-regulated interventions (such as exercise and diet, behavioural, psychological, and physical therapies, and public health interventions) are significantly 
less likely to be registered, ${ }^{12}$ more likely to have inadequate intervention descriptions, ${ }^{26}$ and are not covered by governing bodies able to require that summary results are posted. The priorities outlined in this article apply to all trials of any interventions, not only to trials of drugs, biologics, and devices.

\section{Conclusion}

We believe that calls to make IPD available are usually made to encourage transparency and enable use, reuse, and replication of research. However, these worthy objectives will not be achieved without addressing the problems stemming directly from poor documentation and reporting of research. The potential contribution of IPD will remain unrealised while so much research is poorly reported, irreplicable, and uninterpretable because of selective or deceptive reporting. Trial registries are the only currently feasible mechanism by which the priorities listed in this article can be achieved. Beyond registration of trials, all registries could create fields for summary results to be entered as ClinicalTrials.gov has. Additionally, all trialists could be required to upload protocols and other trial materials. This would make all trial information readily accessible in the one location, as well as enable monitoring and reporting of compliance with providing trial information.

We are strongly supportive of encouraging IPD availability and acknowledge the many examples of its demonstrable value and the efforts that various groups have already made towards enabling data sharing. ${ }^{7}$ However, a more nuanced approach to increasing IPD availability may be required. For example, further work is needed to clarify what is meant by IPD and when and how different data types and different levels of granularity should be made available. ${ }^{10}$

Access to data from research is an important element in improving trials, particularly as permanent data loss occurs rapidly - at about $17 \%$ per year. ${ }^{33}$ Without concurrent work on making the many other elements available, inappropriate emphasis on making IPD available may prove an expensive distraction, and even counterproductive. The priorities identified in this article all offer potentially high yield on investment - they are simple, non-controversial, require minimal changes to existing systems, and some are already required for some categories of trials. Obtaining reliable summary data for all trials offers greater public benefit than obtaining all the data for a minority of trials. 


\section{Key Messages}

- Individual Participant Data (IPD) sharing can be valuable, but it is unhelpful to promote it at the expense of simpler and more cost-efficient measures that are part of the data sharing package and crucial to trial transparency and use.

- Priorities that are more efficient than IPD sharing include requiring and enforcing prospective trial registration, and publication of summary results, protocols, and other trial materials from all controlled trials.

- These priorities apply to trials of any intervention, not just to trials of drugs, biologics, and devices.

- Access to IPD is an important element in improving the information yield from trials, but without concurrent efforts to make the many other elements available for all trials, promoting access to IPD may prove an expensive distraction, or even counterproductive.

Contributors and sources: The authors of this article discussed the latest call for IPD sharing (from the ICJME) and reactions to it from various stakeholders. All of the authors have expertise in the area of minimising research waste. All authors contributed to writing and reviewing the paper. TH is guarantor of the article.

Competing interest: We have read and understood the BMJ Group policy on declaration of interests and declare the following interests: BG receives additional income from speaking and writing for lay audiences about problems in science, including data transparency, and is co-founder of the AllTrials campaign for greater trials transparency. The other authors have no conflicts to declare.

Licence: The Corresponding Author has the right to grant on behalf of all authors and does grant on behalf of all authors, a worldwide licence (http://www.bmj.com/sites/default/files/BMJ\%20Author\%20Licence\%20March\%202013.doc) to the Publishers and its licensees in perpetuity, in all forms, formats and media (whether known now or created in the future), to i) publish, reproduce, distribute, display and store the Contribution, ii) translate the Contribution into other languages, create adaptations, reprints, include within collections and create summaries, extracts and/or, abstracts of the Contribution and convert or allow conversion into any format including without limitation audio, iii) create any other derivative work(s) based in whole or part on the on the Contribution, iv) to exploit all subsidiary rights to 
exploit all subsidiary rights that currently exist or as may exist in the future in the Contribution, v) the inclusion of electronic links from the Contribution to third party material where-ever it may be located; and, vi) licence any third party to do any or all of the above. All research articles will be made available on an Open Access basis (with authors being asked to pay an open access fee-see http://www.bmj.com/about-bmj/resources-authors/forms-policies-and-checklists/copyright-openaccess-and-permission-reuse). The terms of such Open Access shall be governed by a Creative Commons licence - details as to which Creative Commons licence will apply to the research article are set out in our worldwide licence referred to above.

\section{References}

1. Taichman DB, Backus J, Baethge C, Bauchner H, Leeuw PW De, Drazen JM, et al. Sharing clinical trial data. A proposal from the International Committee of Medical Journal Editors. JAMA 2016;315:467-8.

2. Vallance P, Chalmers I. Secure use of individual patient data from clinical trials. Lancet 2013;382:1073-4.

3. Longo DL, Drazen JM. Data sharing. New Engl J Med 2016;374:276-7.

4. Bauchner H, Golub RM, Fontanarosa PB. Data sharing: an ethical and scientific imperative. JAMA 2016;315:1237-9.

5. Strom B, Buyse M, Hughes J, Knoppers B. Data sharing - Is the juice worth the squeeze? New Engl J Med 2016;375:1608-9.

6. Horton R. Offline: Data sharing - why editors may have got it wrong. Lancet 2016;388:1143.

7. Rockhold F, Nisen P, Freeman A. Data sharing at a crossroads. New Engl J Med 2016;375:1115-7.

8. Rosenbaum L. Briding the data-sharing divide - seeing the devil in the details, not the other camp. New Engl J Med 2017; doi: 10.1056/NEJMp1704482.

9. Chalmers I, Glasziou P. Avoidable waste in the production and reporting of research evidence. Lancet 2009;374:86-9.

10. Zarin D, Tse T. Sharing Individual Participant Data (IPD) within the context of the Trial Reporting System (TRS). PLOS Med 2016;13:e1001946.

11. Macleod MR, Michie S, Roberts I, Dirnagl U, Chalmers I, Ioannidis J, et al. Biomedical research : increasing value, reducing waste. Lancet 2014;383:101-4. 
12. Dal-Ré R, Ross JS, Marušić A. Compliance with prospective trial registration guidance remained low in high-impact journals and has implications for primary end point reporting. $J$ Clin Epidemiol 2016;75:100-7.

13. Hooft L, Korevaar DA, Molenaar N, Bossuyt PM, Scholten RJ. Endorsement of ICMJE's clinical trial registration policy: a survey among journal editors. Neth J Med 2014;72:349-55.

14. Dechartres A, Ravaud P, Atal I, Riveros C, Boutron I. Association between trial registration and treatment effect estimates: a meta-epidemiological study. BMC Med 2016;14:100.

15. What does all trials registered and reported mean? [accessed Feb 10 2017]; available from: http://www.alltrials.net/find-out-more/all-trials/

16. World Health Organisation. World Health Organisation Data Set. [accessed Feb 10 2017]; available from: http://www.who.int/ictrp/network/trds/en/

17. Anderson ML, Chiswell K, Peterson ED, Tasneem A, Topping J, Califf RM. Compliance with results reporting at ClinicalTrials.gov. New Engl J Med 2015;372:1031-9.

18. Zarin D, Tse T, Williams R, Carr S. Trial Reporting in ClinicalTrials.gov — The Final Rule. New Engl J Med 2016;375:1998-2004.

19. Tang E, Ravaud P, Riveros C, Perrodeau E, Dechartres A. Comparison of serious adverse events posted at ClinicalTrials.gov and published in corresponding journal articles. BMC Med 2015;13:189.

20. Riveros C, Dechartres A, Perrodeau E, Haneef R, Boutron I, Ravaud P. Timing and completeness of trial results posted at ClinicalTrials.gov and published in journals. PLOS Med 2013;10:e1001566.

21. Pfizer. Disclosure of clinical trial information 2009; [accessed Oct 30 2016]; available from: https://www.pfizer.com/files/research/research_clinical_trials/Disclosure_ClinicalTrialsInfor mation_030209.pdf

22. Batelle Technology Partnership Practice. Biopharmaceutical industry-sponsored clinical trials: impact on state economies. 2015. [accessed Feb 10 2017] Biopharmaceutical industrysponsored clinical trials: impact on state economies. Available from: http://phrmadocs.phrma.org/sites/default/files/pdf/biopharmaceutical-industry-sponsored-clinical-trialsimpact-on-state-economies.pdf

23. Chan A, Tetzlaff JM, Gøtzsche PC, Altman DG, Mann H, Berlin JA, et al. SPIRIT 2013 explanation and elaboration: guidance for protocols of clinical trials. BMJ 2013;346:e7586.

24. Department of Health and Human Services. Clinical trials registration and results information submission. 2016 [accessed Feb 10 2017]; Available from: https://www.federalregister.gov/documents/2016/09/21/2016-22129/clinical-trialsregistration-and-results-information-submission

25. Hoffmann TC, Glasziou PP, Boutron I, Milne R, Perera R, Moher D, et al. Better reporting of interventions: template for intervention description and replication (TIDieR) checklist and guide. BMJ 2014;348:g1687. 
26. Hoffmann TC, Erueti C, Glasziou PP. Poor description of non-pharmacological interventions: analysis of consecutive sample of randomised trials. BMJ 2013;347:f3755.

27. Duff JM, Leather H, Walden EO, LaPlant KD, George TJ. Adequacy of published oncology randomized controlled trials to provide therapeutic details needed for clinical application. $J$ Natl Cancer Inst 2010;102:702-5.

28. Mendel J, Goldacre B, Ernst E, Whittle S. Problems with ethical approval and how to fix them: lessons from three trials in rheumatoid arthritis. BMJ 2016;354:i4626.

29. Goldacre B, Gray J. OpenTrials: towards a collaborative open database of all available information on all clinical trials. Trials 2016;17:164.

30. Doshi P, Jefferson T. Clinical study reports of randomised controlled trials: an exploratory review of previously confidential industry reports. BMJ Open 2013;3:e002496.

31. Weber WEJ, Merino JG, Loder E. Trial registration 10 years on. BMJ 2015;351:h3572.

32. Dal-Ré R, Bracken MB, Ioannidis JP. Call to improve transparency of trials of non-regulated interventions. BMJ 2015;350:h1323.

33. Vines TH, Albert AYK, Andrew RL, Débarre F, Bock DG, Franklin MT, et al. The availability of research data declines rapidly with article age. Curr Biol 2014;24:94-7. 